Pacific Journal of Mathematics

UNIQUENESS FOR THE CAUCHY PROBLEM FOR 


\section{UNIQUENESS FOR THE CAUCHY PROBLEM FOR DEGENERATE PARABOLIC EQUATIONS}

\section{AVNER FrIEDMAN}

Consider a second order degenerate parabolic operator $L$. The present paper is concerned with the uniqueness of solutions of the Cauchy problem: $L u=f$ in a strip $0<t \leqq T$, $u(0, x)=\phi(x)$ for all $x$ in $R^{n}$. It is proved that there is at most one solution subject to a growth condition which depends on the degeneracy of $L$. In the special case where $L$ is ultraparabolic, uniqueness is proved under only onesided growth condition. The methods used involve the construction of comparison functions in suitable sequences of domains.

In $\S 1$ we state the main results on uniqueness of regular solutions. The proofs are given in $\S 2$. In $\S 3$ we derive uniqueness results for weak solutions defined by means of stochastic differential equations. Finally, in $\S 4$, a uniqueness theorem is proved in case $L$ is ultraparabolic, for solutions satisfying only a onesided growth condition.

Results of the same nature as in $\S 1$ were obtained in very special cases in [4], [10]. Results overlapping with those of $\S 3$ have recently been obtained by Sonin [12]; for more details see Remark 1 of $\S 3$.

1. Uniqueness of regular solutions. Let

$$
L u \equiv \sum_{i, j=1}^{n} a_{i j}(t, x) \frac{\partial^{2} u}{\partial x_{i} \partial x_{j}}+\sum_{i=1}^{n} b_{i}(t, x) \frac{\partial u}{\partial x_{i}}+c(t, x) u-\frac{\partial u}{\partial t}
$$

where $\left(a_{i j}\right)$ is a symmetric positive semidefinite matrix. Let $k_{i}$ $(1 \leqq i \leqq p)$ and $m$ be positive intergers such that $1 \leqq k_{1}<k_{2}<\cdots<k_{p}=$ $m \leqq n$, and set

$$
A_{j}=\left\{k_{j-1}+1, k_{j-1}+2, \cdots, k_{j}\right\}, 1 \leqq j \leqq p, \text { where } k_{0}=0 .
$$

Write

$$
x=\left(x^{\prime}, x^{\prime \prime}\right), x^{\prime}=\left(x_{1}^{\prime}, \cdots, x_{p}^{\prime}\right), x_{j}^{\prime}=\left(x_{k_{j-1}+1}, \cdots, x_{k_{j}}\right) .
$$

Let $d_{i}(r) \quad(1 \leqq i \leqq p)$ be positive value functions for $r \geqq 0$, having two continuous derivatives in $\sqrt{r}$ and satisfying

$$
\left\{\begin{array}{l}
\left|\frac{r d_{i}^{\prime}(r)}{d_{i}(r)}\right| \leqq C,\left(d_{i}(r)\right)^{2}\left|\frac{d}{d r} \frac{d_{i}^{\prime}(r)}{\left(d_{i}(r)\right)^{2}}\right| \leqq C . \\
\frac{r}{d_{i}(r)} \text { is monotone increasing in } r(1 \leqq i \leqq p) .
\end{array}\right.
$$


Here and in what follows, various positive constants will be denoted by the same symbol $C$. Note that (1.1) holds if

$$
d_{i}(r)=\left(1+r^{2}\right)^{\rho_{i}}, \quad \rho_{i} \leqq \frac{1}{2} .
$$

We shall assume:

$$
\begin{aligned}
& a_{i i}(t, x) \leqq C d_{k}\left(\left|x_{k}^{\prime}\right|\right) \text { if } i \in A_{k}, 1 \leqq k \leqq p, \\
& a_{i j}(t, x)=0 \text { if } m+1 \leqq i \leqq n, 1 \leqq j \leqq n,
\end{aligned}
$$$$
\left|b_{i}(t, x)\right| \leqq C\left(1+\left|x_{k}^{\prime}\right|+\sum_{l \neq k}\left|x_{l}^{\prime}\right|^{\mu_{l k}}+C\left|x^{\prime \prime}\right|^{r_{k}}\right)
$$$$
\text { if } i \in A_{k}, 1 \leqq k \leqq p \text {, }
$$

$$
\left|b_{j}(t, x)\right| \leqq C\left(1+\sum_{k=1}^{p}\left|x_{k}^{\prime}\right|^{\delta_{k}}+\left|x^{\prime \prime}\right|\right) \quad \text { if } m+1 \leqq j \leqq n,
$$

$$
c(t, x) \leqq C\left(1+\sum_{k=1}^{p} \frac{\left|x_{k}^{\prime}\right|^{2}}{d_{k}\left(\left|x_{k}^{\prime}\right|\right)}+\left|x^{\prime \prime}\right|^{2}\right)
$$

where $\mu_{l k}, \gamma_{k}, \delta_{k}, \lambda$ are nonnegative numbers subject to the following conditions:

$$
\begin{gathered}
r^{2 \mu_{k l} l} d_{l}(r) \leqq C\left(1+r^{2}\right) d_{k}\left(r^{\mu k l}\right), \\
r^{2 \gamma_{k}} \leqq C\left(1+r^{2}\right) d_{k}\left(r^{\gamma k}\right), \\
d_{k}(r) \leqq C\left(1+r^{2-} \delta_{k}\right), d_{k}(r) \leqq C\left(1+r^{2-\lambda \delta_{k}}\right)
\end{gathered}
$$

If

$$
\frac{r^{2}}{d_{i}(r)}=R^{2} \text {, then write } r=e_{i}(R) \text {. }
$$

(By (1.1), $e_{i}(R)$ is uniquely defined.) Set

$$
e(R)=\max _{1 \leqq i \leqq p}\left[e_{i}(R)\right]^{\delta_{i}} .
$$

A function $u(t, x)$ will be called regular if it is continuous in $[0, T] \times R^{n}$ and if its partial derivatives $u_{t}, u_{x}, u_{x x}$ exist in $(0, T] \times R^{n}$.

Theorem 1. Assume that (1.3)-(1.7) and (1.1), (1.8)-(1.10) hold. Let $u(t, x)$ be a regular function satisfying

$$
\begin{aligned}
L u(t, x) & =0 \text { in }(0, T] \times R^{n}, \\
u(0, x) & =0 \text { in } R^{n},
\end{aligned}
$$




$$
|u(t, x)| \leqq C \exp \left\{\beta\left[\sum_{k=1}^{p} \frac{\left|x_{k}^{\prime}\right|^{2}}{d_{k}\left(\left|x_{k}^{\prime}\right|\right)}+\left|x^{\prime \prime}\right|^{\lambda}\right]\right\}
$$

in $[0, T] \times R^{n}$, provided

$$
\left|x^{\prime \prime}\right| \leqq e(R), \text { where } R^{2}=\sum_{k=1}^{p} \frac{\left|x_{k}^{\prime}\right|^{2}}{d_{k}\left(\left|x_{k}^{\prime}\right|\right)}
$$

Then $u(t, x) \equiv 0$ in $[0, T] \times R^{n}$.

Stronger assertions can be made in case the $\delta_{k}$ vanish. Suppose

$$
\begin{aligned}
\left|b_{i}(t, x)\right| \leqq C\left(1+\left|x_{k}^{\prime}\right|+\sum_{l \neq k}\left|x_{l}^{\prime}\right|^{\mu_{k l}}\right) \gamma\left(t, x^{\prime \prime}\right) \quad(1 \leqq i \leqq m), & (m+1 \leqq j \leqq n), \\
\left|b_{j}(t, x)\right| \leqq C\left(1+\left|x^{\prime \prime}\right|\right) \quad(m+1) & \leqq C\left(1+\sum_{k=1}^{p} \frac{\left|x_{k}^{\prime}\right|^{2}}{d_{k}\left(\left|x_{k}^{\prime}\right|\right)}\right) \gamma\left(t, x^{\prime \prime}\right)
\end{aligned}
$$

where $\gamma\left(t, x^{\prime \prime}\right)$ is an arbitrary continuous function. Then we can state:

Theorem 2. Assume that (1.3), (1.4), (1.15)-(1.17), and (1.1), (1.8) hold. Let $u(t, x)$ be a regular function satisfying (1.12), (1.13), and let

$$
|u(t, x)| \leqq C \exp \left\{\beta \sum_{k=1}^{p} \frac{\left|x_{k}^{\prime}\right|^{2}}{d_{k}\left(\left|x_{k}^{\prime}\right|\right)}\right\} \mu\left(t, x^{\prime \prime}\right)
$$

where $\mu\left(t, x^{\prime \prime}\right)$ is a continuous function. Then $u(t, x) \equiv 0$ in $[0, T] \times R^{n}$.

2. Proofs of Theorem 1.2. The proofs of Theorems 1, 2 are based on the construction of (i) a comparison function $H(t, x)$, and (ii) a suitable family of increasing domains $D_{R}, R>0$. We begin with the proof of Theorem 1 . We take

$$
H(t, x)=\exp \left\{\frac{k}{1-\mu t}\left[\sum_{k=1}^{p} \frac{\left|x_{k}^{\prime}\right|^{2}}{d_{k}\left(\left|x_{k}^{\prime}\right|\right)}+\alpha\left(x^{\prime \prime}\right)\right]+\nu t\right\}
$$

where $k>2 \beta$ and

$$
\alpha\left(x^{\prime \prime}\right)=\left(1+\left|x^{\prime \prime}\right|^{2}\right)^{\lambda / 2} .
$$

By direct computations one finds that

$$
L H(t, x)<0
$$

provided $\mu, \nu$ are positive and sufficiently large, and 


$$
0 \leqq t \leqq \frac{1}{2 \mu}
$$

Here we make use of (1.3)-(1.7), (1.1), (1.8)-(1.10), and the inequality

$$
\left|a_{i j}\right| \leqq \sqrt{a_{i i}} \sqrt{a_{j j}} \text {. }
$$

We shall take $D_{R}$ to be a product $D_{R}^{\prime} \times D_{R}^{\prime \prime}$ where

$$
D_{R}^{\prime}=\left\{x^{\prime} ; \sum_{k=1}^{p} \frac{\left|x_{k}^{\prime}\right|^{2}}{d_{k}\left(\left|x_{k}^{\prime}\right|\right)}<R^{2}\right\}
$$

and $D_{R}^{\prime \prime}$ the interior of a cone in the $\left(t, x^{\prime \prime}\right)$-space with base

$$
S_{\rho}=\left\{\left(t, x^{\prime \prime}\right) ; t=0,\left|x^{\prime \prime}\right|<\rho\right\}
$$

and vertex $(\eta, 0)$. Clearly

$$
\partial D_{R}=\left[\partial D_{R}^{\prime} \times \overline{D_{R}^{\prime \prime}}\right] \cup\left[D_{R}^{\prime} \times \partial D_{R}^{\prime \prime}\right]
$$

where $\partial \Omega, \bar{\Omega}$ denote respectively the boundary and closure of a set $\Omega$. Also, $\partial D_{R}^{\prime \prime}=S_{\rho} \cup \Gamma$ where $\Gamma$ is spanned by the generators of the cone. Note that

$$
\begin{aligned}
\left|b_{j}(t, x)\right| \leqq & C\left(1+e(R)+\left|x^{\prime \prime}\right|\right) \leqq C(1+e(R)+\rho) \\
& \text { if } m+1 \leqq j \leqq n,(t, x) \in D_{R}^{\prime} \times \Gamma .
\end{aligned}
$$

We wish to choose $\rho=\rho(R)$, $\eta$ such that the partial derivative

$$
-\frac{\partial w}{\partial t}+\sum_{j=m+1}^{n} b_{j}\left(t, x^{\prime}, x^{\prime \prime}\right) \frac{\partial w}{\partial x_{j}}
$$

at the points of $D_{R}^{\prime} \times \Gamma$ is a derivative in a direction pointing into $D_{R}$. This direction is determined by the trajectories

$$
\left\{\begin{array}{l}
\frac{d t}{d s}=-1, \\
\frac{d x_{j}}{d s}=b_{j}\left(t, x^{\prime}, x^{\prime \prime}\right) \quad(m+1 \leqq j \leqq n)
\end{array}\right.
$$

initiating on $D_{R}^{\prime} \times \Gamma$. Thus we want to choose $\rho=\rho(R)$, $\eta$ so that

$$
\frac{d}{d s}\left\{\left[\sum_{j=m+1}^{n} x_{j}^{2}(s)\right]^{1 / 2}-\frac{\rho}{\eta}(\eta-t)\right\}<0
$$

at the points $s=s_{0}$ where $\sum_{j=m+1}^{n} x_{j}^{2}(s)=\left(\rho^{2} / \eta^{2}\right)(\eta-t)^{2}$. Since, by (2.2), (2.4),

$$
\left\{\sum_{j=m+1}^{n}\left(\frac{d x_{i}}{d s}\right)^{2}\right\}^{1 / 2} \leqq C(1+e(R)+\rho)
$$


at $s=s_{0}$, it suffices to choose $\rho$, $\eta$ such that

$$
C(1+e(R)+\rho)<\frac{\rho}{\eta}
$$

\section{Taking}

$$
\rho=e(R), \quad \eta<\frac{1}{3 C},
$$

(2.5) is satisfied for all sufficiently large $R$. We further restrict $\eta$ by: $\eta \leqq 1 /(2 \mu)$.

Consider now, for any $\varepsilon>0$, the function

$$
v(t, x)=[\varepsilon H(t, x) \pm u(t, x)] e^{\gamma t}
$$

in $\bar{D}_{R}$, where $\gamma \geqq c(t, x)$ in $\bar{D}_{R}$. It satisfies

$$
(L-\gamma) v(t, x)<0 \text { in } \bar{D}_{R} \cap\{t>0\} \text {. }
$$

The function $v$ cannot assume a negative minimum at points of $D_{R}$, for at such points $(L-\gamma) v(t, x) \geqq 0$. Next, $v$ cannot attain a negative minimum in $\bar{D}_{R}$ at points $D_{R}^{\prime} \times \Gamma$, for at such points

$$
-\frac{\partial v}{\partial t}+\sum_{j=m+1}^{n} b_{j}(t, x) \frac{\partial v}{\partial x_{j}} \geqq 0
$$

by the choice of $\rho, \eta$ in (2.6), and

$$
\sum_{i, j=1}^{m} a_{i j}(t, x) \frac{\partial^{2} v}{\partial x_{i} \partial x_{j}} \geqq 0, \frac{\partial v}{\partial x_{i}}=0 \quad(1 \leqq i \leqq m),
$$

so that again $(L-\gamma) v \geqq 0$, which is impossible.

Since $v>0$ on $D_{R}^{\prime} \times S_{\rho}$ (by (1.13)) and $v>0$ on $\partial D_{R}^{\prime} \times D_{R}^{\prime \prime}$ if $R$ is large (by (1.14)), we conclude that $v>0$ in $D_{R}$. Hence

$$
|u(t, x)| \leqq \varepsilon H(t, x)
$$

at each point $(t, x)$ in $D_{R}$. Taking $R \rightarrow \infty$ and noting that each point $(t, x)$ with $0<t<\eta$ is contained in $D_{R}$ if $R$ is sufficiently large, it follows that (2.7) holds in the whole strip $0<t<\eta$. Taking $\varepsilon \rightarrow 0$ we conclude that $u(t, x) \equiv 0$ in the strip $0 \leqq t \leqq \eta$. We can now proceed step by step to prove that $u(t, x) \equiv 0$ in the strip $0 \leqq t \leqq T$.

To prove Theorem 2 we note that (2.5) now becomes

$$
C(1+\rho)<\frac{\rho}{\eta}
$$

We take $\rho$ a fixed positive number $>1$ and independent of $R$, and 


$$
\eta<\frac{1}{2 C}
$$

We also take $\alpha\left(x^{\prime \prime}\right) \equiv 0$ in the definition of $H(t, x)$. Then, if $\mu, \nu$ are sufficiently large (depending on $\rho$ ) and $\eta \leqq 1 /(2 \mu)$, then $L H<0$ in $\bar{D}_{R}$. We can now proceed as before to establish the inequality

$$
|u(t, x)| \leqq \varepsilon H\left(t, x^{\prime}\right)
$$

in $D_{R}$. Taking $R \rightarrow \infty$ ( $\rho$ is fixed) and then $\varepsilon \rightarrow 0$, we conclude that

$$
u\left(t, x^{\prime}, x^{\prime \prime}\right)=0 \text { if } x^{\prime} \in R^{m},\left(t, x^{\prime \prime}\right) \in D_{R}^{\prime \prime} ;
$$

the cone $D_{R}^{\prime \prime}$ is independent of $\rho$. Since this is true for any cone $D_{R}^{\prime \prime}$ with base $S_{\rho}, u(t, x) \equiv 0$ in the strip $0 \leqq t \leqq \eta$. A step-by-step argument gives $u(t, x) \equiv 0$ in the strip $0 \leqq t \leqq T$.

REMARK 1. If the $d_{i}(r)$ are given by (1.2), then (1.8)-(1.10) reduce to

$$
\begin{gathered}
\rho_{l}+2 \mu_{k l} \leqq 2+\mu_{k l} \rho_{k}, \quad\left(2+\rho_{k}\right) \gamma_{k} \leqq \lambda, \\
\lambda \delta_{k} \leqq 2+\rho_{k}, \quad \delta_{k} \leqq 2+\rho_{k} .
\end{gathered}
$$

We can take

$$
e(R)=C \max _{m+1 \leqq i \leqq n}\left\{1+R^{2 \tilde{o}_{i} /\left(2+\rho_{i}\right)}\right\}
$$

REMARK 2. Using different comparison functions for $H$ (such as

$$
\left[\sum_{k=1}^{p} \frac{\left|x_{k}^{\prime}\right|^{2}}{d_{k}\left(\left|x_{k}^{\prime}\right|\right)}+K t\right]^{q} e^{\alpha t} \quad(K, q, \alpha \text { positive })
$$

cf. [3; p. 56]) one can obtain variants of Theorems 1,2 where the growth conditions on $a_{i j}, b_{i}, c$ and on the solution $u$ are modified.

3. Uniqueness for weak solutions. We assume

(A) The coefficients $a_{i j}, b_{i}$ are independent of $t$ and $c(t, x) \equiv 0$.

(B) There is an $m \times m$ matrix $\sigma(x)=\left(\sigma_{i j}(x)\right)$ such that

$$
2 a_{i j}(x)=\sum_{k=1}^{m} \sigma_{i k}(x) \sigma_{j k}(x) \quad(1 \leqq i, j \leqq m)
$$

and the functions $\sigma_{i k}(x), b_{j}(x)(1 \leqq i, k \leqq m, 1 \leqq j \leqq n)$ are Lipschitz continuous in $x$, uniformly in compact subsets of $R^{n}$.

$$
\sum_{i, j=1}^{m}\left|\sigma_{i j}(x)\right|+\sum_{i=1}^{n}\left|b_{i}(x)\right| \leqq C(1+|x|) .
$$

Recall [2], [9] that if (1.4) holds and the $a_{i j}(x)$ have continuous 
second derivatives then there exists a matrix $\sigma(x)$ satisfying (B).

Consider the stochastic differential system (see [5], [6] for the relevant theory)

$$
\begin{array}{lr}
d \xi_{i}(t)=b_{i}(\xi(t)) d t+\sum_{j=1}^{m} \sigma_{i j}(\xi(t)) d w_{j}(t) & (1 \leqq i \leqq m), \\
d \xi_{j}(t)=b_{j}(\xi(t)) d t & (m+1 \leqq j \leqq n)
\end{array}
$$

where the $w_{j}(t)$ are independent Brownian motions. Let $G$ be any bounded domain in $R^{n}$ and denote by $\tau$ the exit time of $(s, \xi(s))$ from the cylinder $[0, t) \times G$, where $\xi(s)(0 \leqq s \leqq t)$ is the solution of (3.1) with $\xi(0)=x$.

Definition. A continuous function $u(t, x)$ in the strip $[0, T] \times R^{n}$ is a weak solution of the equation $L u=0$ if for any bounded domain $G$ in $R^{n}$ and for every $t \in(0, T]$,

$$
u(t, x)=E_{x} u(t-\tau, \xi(\tau)) .
$$

A slightly different definition was used in [12]. If $u$ is a classical solution $L u=0$ in the strip $(0, T]$, continuous in $[0, T]$ then, by Ito's formula, it is a weak solution. Conversely, a smooth weak solution is a classical solution. A weak solution satisfying $u(0, x)=\phi(x)$ in $R^{n}$ is called a weak solution of the Cauchy problem

$$
\begin{aligned}
L u(t, x) & =0 \text { in }(0, T] \times R^{n}, \\
u(0, x) & =\phi(x) \text { in } R^{n} .
\end{aligned}
$$

If $H$ is a smooth function satisfying $L H \leqq 0$ in the strip [0,T] then, by Ito's formula,

$$
H(t, x) \geqq E_{x} H(t-\tau, \xi(\tau)) .
$$

From this and (3.2) we obtain, for any $\varepsilon>0$,

$$
\varepsilon H(t, x) \pm u(t, x) \geqq E_{x}\{\varepsilon H(t-\tau, \xi(\tau)) \pm u(t-\tau, \xi(\tau))\} .
$$

Suppose now that the conditions of Theorem 1 are satisfied. Modify the definition of the domain $D_{R}=D_{R}^{\prime} \times D_{R}^{\prime \prime}$ used in the proof of Theorem 1, by taking

$$
D_{R}^{\prime \prime}=\left\{\left(t, x^{\prime \prime}\right) ; 0<t<T,\left|x^{\prime \prime}\right|<\rho\right\} .
$$

Denote by $\tau$ the exit time of $(s, \xi(s))$ from the cylinder $D_{R} \cap\{s<t\}$; $t \leqq \eta$. For $m+1 \leqq i \leqq n, 0<s<\tau$, 


$$
\begin{aligned}
\left|\frac{d \xi_{i}(s)}{d s}\right| & =\left|b_{i}(s, \xi(s))\right| \leqq C\left(1+\sum_{k=1}^{p}\left|\xi_{k}^{\prime}(s)\right|^{\delta_{k}}+\sum_{i=m+1}^{n}\left|\xi_{i}(s)\right|\right) \\
& \leqq C(1+e(R)+\rho) .
\end{aligned}
$$

Hence, if $\xi(0)=x^{0}$ is fixed, $R$ is large and $\rho=e(R), \eta<1 /(3 C)$, then $\xi^{\prime \prime}(s)=\left(\xi_{m+1}(s), \cdots, \xi_{n}(s)\right)$ does not leave the ball $\left|x^{\prime \prime}\right|<\rho$ at $s=\tau$. Next, if $u(0, x) \equiv 0$ then $u(t-\tau, \xi(\tau))=0$ if $\tau=t$. Using (3.4) with the same $H$ as in the proof of Theorem 1, we conclude that

$$
\varepsilon H\left(t, x^{0}\right) \pm u\left(t, x^{0}\right) \geqq E_{x^{0}}\{\chi[\varepsilon H(t-\tau, \xi(\tau)) \pm u(t-\tau, \xi(\tau))]\}
$$

where $\chi=1$ if $\xi(\tau)$ lies on $\partial D_{R}^{\prime} \times D_{R}^{\prime \prime}$ and $\tau<t$, and $\chi=0$ otherwise. Since on the set where $\chi=1$,

$$
(\varepsilon H \pm u) \longrightarrow 0 \text { if } R \longrightarrow \infty,
$$

it follows that $\left|u\left(t, x^{0}\right)\right| \leqq \varepsilon H\left(t, x^{0}\right)$. Taking $\varepsilon \rightarrow 0$ we conclude that $u\left(t, x^{0}\right)=0$. Hence $u(t, x) \equiv 0$ in the strip $0 \leqq t \leqq \eta$. We thus obtain:

THEOREM 3. Let the conditions (A)-(C), (1.3)-(1.6), (1.8)-(1.10) hold. If $u(t, x)$ is a weak solution of the Cauchy problem (1.12), (1.13) and if it satisfies (1.14) where $\left|x^{\prime \prime}\right| \leqq e(R) \quad(R, e(R)$ as in Theorem 1), then $u(t, x) \equiv 0$ in $[0, T] \times R^{n}$.

\section{Similarly one can prove:}

THEOREM 4. Let the conditions (A)-(C), (1.3), (1.4), (1.15), (1.16), and (1.1), (1.8) hold. If $u(t, x)$ is a weak solution of the Cauchy problem (1.12), (1.13) and if it satisfies (1.18) where $\mu\left(t, x^{\prime \prime}\right)$ is a continuous function, then $u(t, x) \equiv 0$ in $[0, T] \times R^{n}$.

Split the coordinates of $x^{\prime \prime}$ into $q$ sets:

$$
x^{\prime \prime}=\left(x_{1}^{\prime \prime}, \cdots, x_{q}^{\prime \prime}\right), \quad x_{j}^{\prime \prime}=\left(x_{\sigma_{j-1}+1}^{\prime \prime}, \cdots, x_{\sigma_{j}}^{\prime \prime}\right) \quad(1 \leqq j \leqq q)
$$

where $\sigma_{0}=m+1<\sigma_{1}<\cdots<\sigma_{q}=n$, and let $B_{j}=\left\{\sigma_{j-1}+1, \cdots, \sigma_{j}\right\}$. We can refine Theorems $1-4$ by imposing different growth conditions on the $b_{i}$ with respect to the variable $x_{j}^{\prime \prime}$. We give here one example.

Suppose

$$
\begin{array}{cc}
a_{i i}(x) \leqq C & (1 \leqq i \leqq m), \\
\left|b_{i}(x)\right| \leqq C\left(1+\left|x^{\prime}\right|+\left|x^{\prime \prime}\right| r\right) & (1 \leqq i \leqq m),
\end{array}
$$

and (1.4) holds, and replace (1.6) by 


$$
\left|b_{j}(x)\right| \leqq C\left(1+\left|x^{\prime}\right|^{\delta} l+\left|x^{\prime \prime}\right|\right) \text { if } j \in B_{l}, 1 \leqq l \leqq q .
$$

We can then proceed as in the proof of Theorem 3, but with modified $D_{R}=D_{R}^{\prime} \times D_{R}^{\prime \prime}$ :

$$
\begin{gathered}
D_{R}^{\prime}=\left\{x^{\prime} ;\left|x^{\prime}\right|<R\right\} \\
D_{R}^{\prime \prime}=D_{R, 1} \times \cdots \times D_{R, q}, \quad D_{R, j}=\left\{x_{j}^{\prime \prime} ;\left|x_{j}^{\prime \prime}\right|<R^{\delta}\right\} .
\end{gathered}
$$

We also take a modified function $H$ :

$$
H(t, x)=\exp \left\{\frac{k}{1-\mu t}\left[\left|x^{\prime}\right|^{2}+\sum_{l=1}^{q}\left(1+\left|x_{l}^{\prime \prime}\right|^{2}\right)^{\lambda_{l} / 2}\right]+\nu t\right\}
$$

where $\lambda_{l}=2 / \delta_{l}$. If

$$
\gamma \leqq \min _{1 \leqq l \leqq q} \frac{1}{\delta_{l}}
$$

then we can again prove that $L H<0$ provided $\mu, \nu$ are sufficiently large and $0 \leqq t \leqq 1 /(2 \mu)$. We sum up:

THEOREM 5. Let the conditions (A)-(C), (1.4) and (3.5)-(3.8) hold. If $u$ is a weak solution of (1.12), (1.13) and

$$
|u(t, x)| \leqq C \exp \left\{\beta\left[\left|x^{\prime}\right|^{2}+\sum_{l=1}^{q}\left|x_{l}^{\prime \prime}\right|^{2 / \hat{o}_{l}}\right]\right\}
$$

then $u(t, x) \equiv 0$ in $[0, T] \times R^{n}$.

REMARK 1. Sonin [12] has proved theorems which overlap with Theorems 3-5. His method is entirely probabilistic; our method is much simpler. In the growth condition on $u(t, x)$ he allows $\left|x^{\prime}\right|^{2}$ to be replaced by a slightly more general function, namely, $\left|x^{\prime}\right| h\left(\left|x^{\prime}\right|\right)$ where

$$
\int^{\infty} \frac{d r}{h(r)}=\infty
$$

However he imposes more restrictive growth conditions on the $b_{i}(t, x)$ $(1 \leqq i \leqq n)$.

REMARK 2. Under some smoothness and growth conditions on $\phi(x)$, one can easily establish the existence of a regular solution for the Cauchy problem (3.3) (with coefficients depending also on $t$ ) by means of a probabilistic formula. It is convenient to write the Cauchy problem in the form 


$$
\begin{array}{r}
\frac{\partial v}{\partial t}+\sum_{i, j=1}^{n} a_{i j}(t, x) \frac{\partial^{2} v}{\partial x_{i} \partial x_{j}}+\sum_{i=1}^{n} b_{i}(t, x) \frac{\partial v}{\partial x_{i}}+c(t, x) v=0 \\
\text { in }[0, T) \times R^{n},
\end{array}
$$

$$
v(T, x)=\phi(x) \text { in } R^{n} .
$$

This form is obtained from (3.3) by the transformation $t=T-s$ upon denoting $a_{i j}(T-s, x), b_{i}(T-s, x), c(T-s, x)$ by $a_{i j}(s, x), b_{i}(s, x)$, $c(s, x)$ respectively. Suppose there is a matrix $\left(\sigma_{i j}(t, x)\right)(i, j=1, \cdots, m)$ such that

$$
2 a_{i j}(t, x)=\sum_{k=1}^{m} \sigma_{i k}(t, x) \sigma_{j k}(t, x) \quad(1 \leqq i, j \leqq m),
$$

and suppose $\sigma_{i j}(t, x), b_{l}(t, x)$ satisfy a uniform Lipschitz condition in $t, x$ and are bounded by $C(1+|x|)$. Denote by $\xi_{x, t}(s)$ the solution of

$$
\begin{aligned}
d \xi_{i}(s) & =b_{i}(s, \xi(s)) d s+\sum_{j=1}^{m} \sigma_{i j}(s, \xi(s)) d w_{j}(s) & (1 \leqq i \leqq m), \\
d \xi_{k}(s) & =b_{k}(s, \xi(s)) d s & (m+1 \leqq k \leqq n), \\
\xi_{x, t}(t) & =x . &
\end{aligned}
$$

Let

$$
v(t, x)=E_{x}\left\{\phi\left(\xi_{x, t}(T)\right) \exp \left[\int_{t}^{T} c\left(s, \xi_{x, t}(s)\right) d s\right]\right\} \cdot
$$

If

$$
\left|D_{x}^{i} \phi(x)\right| \leqq C\left(1+|x|^{2 r}\right) \text { for } i=0,1,2(r>0),
$$

if $c(s, x) \leqq 0$, and if the derivatives

$$
D_{t} \sigma_{i j}, D_{t} b_{i}, D_{x}^{\alpha} \sigma_{i j}, D_{x}^{\alpha} b_{i}, D_{x}^{\alpha} c \quad(\alpha=1,2)
$$

are continuous and bounded then, by [6], $v(t, x)$ is a regular solution of (3.10), and $|v(t, x)| \leqq C\left(1+|x|^{r}\right)$.

If $m<n$ and if $\delta_{k}=0$ in the condition (1.6), then the last assertion is valid under weaker growth conditions on $\phi(x)$, namely, (3.12) may be replaced by

$$
\begin{aligned}
& \left|\phi\left(x^{\prime}, x^{\prime \prime}\right)\right|+\left|D_{x} \phi\left(x^{\prime}, x^{\prime \prime}\right)\right|+\left|D_{x x} \phi\left(x^{\prime}, x^{\prime \prime}\right)\right| \\
\leqq & C\left(1+\left|x^{\prime}\right| r\right) g\left(x^{\prime \prime}\right)
\end{aligned}
$$

where $g\left(x^{\prime \prime}\right)$ is a continuous function. The regular solution $v(t, x)$ is bounded by $C\left(1+\left|x^{\prime}\right|^{r}\right) h\left(x^{\prime \prime}\right), h\left(x^{\prime \prime}\right)$ a continuous function.

REMARK 3. Suppose (A)-(C) hold and suppose $\sigma_{i j}(x), b_{k}(x)$ satisfy a uniform Lipschitz condition in $R^{n}$. If $\phi(x)$ is a continuous function satisfying 


$$
|\phi(x)| \leqq C\left(1+|x|^{r}\right)
$$

then the function

$$
u(t, x)=E_{x}\{\phi(\xi(t))\}
$$

is well defined. Using the Strong Markov property of the solutions of (3.1) one can easily show that $u$ is a weak solution of the Cauchy problem (3.3). If $m<n$ and $\delta_{k}=0$ in (1.6), then the last assertion remains true if (3.14) is replaced by

$$
|\phi(x)| \leqq C\left(1+\left|x^{\prime}\right|^{r}\right) g\left(x^{\prime \prime}\right)
$$

where $g\left(x^{\prime \prime}\right)$ is a continuous function.

4. Uniqueness for solutions satisfying a lower growth condition. From the proof of Theorem 1 we obtain the following maximum-principle-type result:

Lemma 1. Assume that (1.3)-(1.7) and (1.6), (1.8)-(1.10) hold. Let $u(t, x)$ be a regular function satisfying

$$
\begin{aligned}
L u(t, x) & \leqq 0 \text { in }(0, T] \times R^{n}, \\
u(0, x) & \geqq 0 \text { in } R^{n}, \\
u(t, x) & \geqq-C \exp \left\{\beta\left[\sum_{k=1}^{p} \frac{\left|x_{k}^{\prime}\right|^{2}}{d_{k}\left(\left|x_{k}^{\prime}\right|\right)}+\left|x^{\prime \prime}\right|^{\lambda}\right]\right\}
\end{aligned}
$$

in $[0, T] \times R^{n}$ provided

$$
\left|x^{\prime \prime}\right| \leqq e(R), \text { where } R^{2}=\sum_{k=1}^{p} \frac{\left|x_{k}^{\prime}\right|^{2}}{d_{k}\left(\left|x_{k}^{\prime}\right|\right)},
$$

where $C, \beta$ are positive constants. Then $u(t, x) \geqq 0$ in $[0, T] \times R^{n}$.

Similar results hold under the assumptions of Theorems 2-5.

We shall prove in this section that, under further restrictions on $L$, if $L u=0$ in $0<t \leqq T, u(0, x)=0$ on $R^{n}, u(t, x) \geqq 0$ in $0 \leqq t \leqq T$, then $u(t, x)=0$ in $0 \leqq t \leqq T$. For such results for nondegenerate parabolic equations, see Friedman [3], Aronson and Besala [1] and the references given there.

We shall take $L$ to have the form

$$
\begin{gathered}
L u \equiv \sum_{i, j=1}^{m} a_{i j}(t, x, y) \frac{\partial^{2} u}{\partial x_{i} \partial x_{j}}+\sum_{i=1}^{m} a_{i}(t, x, y) \frac{\partial u}{\partial x_{i}} \\
+\sum_{i=1}^{k} x_{i} \frac{\partial u}{\partial y_{i}}+c(t, x, y) u-\frac{\partial u}{\partial t}
\end{gathered}
$$


where $k \leqq m$ and the matrix $\left(a_{i j}\right)$ is uniformly positive definite. Such operators are called ultraparabolic operators. If the coefficients are sufficiently regular, then (see Weber [13], Ilin [7], Sonin [11]) there exists a fundamental solution $\Gamma(t, x, y ; \tau, \xi, \eta)$ for the Cauchy problem

$$
\begin{gathered}
L u=0 \text { in } 0<t \leqq T, \\
u(0, x, y)=\phi(x, y) \text { for }(x, y) \in R^{m+k} .
\end{gathered}
$$

Thus, if $\phi$ is continuous and bounded, then a solution of (4.2), (4.3) is given by

$$
u(t, x, y)=\int_{R^{k}} \int_{R^{m}} \Gamma(t, x, y ; 0, \xi, \eta) \phi(\xi, \eta) d \xi d \eta
$$

Set

$$
\Pi=\left\{(t, x, y) ; 0 \leqq t \leqq T, x \in R^{m}, y \in R^{k}\right\} .
$$

We shall assume:

$(P)$ There exists a positive constant $\alpha_{0}$ such that

$$
\sum_{i, j=1}^{m} a_{i j}(t, x, y) \xi_{i} \xi_{j} \geqq \alpha_{0}|\xi|^{2} \text { for all } \xi \in R^{m},(t, x, y) \in \Pi \text {. }
$$

(Q) The functions $a_{i j}$ and their first three derivatives are continuous and bounded in $\Pi$; the functions $a_{i}$ and their first two derivatives are continuous and bounded in $\Pi$; the function $c$ and its first derivatives are continuous and bounded in $I I$.

Under these assumptions the above mentioned fundamental solution exists and, for any $K>0, c>0$,

$$
\begin{aligned}
& |\Gamma(t, x, y ; \tau, \xi, \eta)|+\sum_{i=1}^{m}\left|\frac{\partial}{\partial x_{i}} \Gamma(t, x, y ; \tau, \xi, \eta)\right| \\
\leqq & M \exp \left\{-\mu|x|^{2}-\mu\left|x^{\prime}+y\right|^{2}\right\} \quad(M>0, \mu>0)
\end{aligned}
$$

where $x^{\prime}=\left(x_{1}, \cdots, x_{k}\right)$, provided $|\xi|+|\eta| \leqq K, t-\tau \geqq c$, where $M, \mu$ depend on $K, c$. [Sharper estimates are valid (see [7]) but will not be needed here.] Furthermore, for the adjoint Cauchy problem

$$
\begin{aligned}
L^{*} v(\tau, \xi, \eta) & =0 \text { in } 0 \leqq \tau<T, \\
v(T, \xi, \eta) & =\psi(\xi, \eta) \text { for }(\xi, \eta) \in R^{m+k}
\end{aligned}
$$

there also exists a fundamental solution $\Gamma^{*}(\tau, \xi, \eta ; \bar{\tau}, \bar{\xi}, \bar{\eta})(\tau<\bar{\tau})$. Using Green's identity as in [3; p. 29] and employing (4.4) and the analogous estimates for $\Gamma^{*}$, one deduces that

$$
\Gamma(t, x, y ; \tau, \xi, \eta)=\Gamma^{*}(\tau, \xi, \eta ; t, x, y) .
$$


Hence,

$$
L^{*} \Gamma(t, x, y ; \tau, \xi, \eta)=0 \text { for each fixed }(t, x, y) \text {. }
$$

Note that $\Gamma \geqq 0$; the proof is as in the nondegenerate parabolic case $[3 ; \mathrm{p} .45]$.

Theorem 6. Let $(P),(Q)$ hold and let $u(t, x)$ be a regular solution of (4.2) satisfying

$$
u(t, x, y) \geqq-C \exp \left\{\beta\left[|x|^{2}+|y|^{2}\right]\right\} \quad(C>0, \beta>0)
$$

provided $|y| \leqq|x|$. If $u(0, x, y)=0$ on $R^{m+k}$ then $u(t, x, y) \equiv 0$ in $\Pi$.

Proof. In view of Lemma 1 it suffices to prove the theorem in case $u(t, x, y) \geqq 0$ in $\Pi$.

If $k<m$, then introduce

$$
\begin{gathered}
\widetilde{\Pi}=\left\{\left(t, x, y, y_{k+1}, \cdots, y_{m}\right) ; 0<t<T,\left(x, y, y_{k+1}, \cdots, y_{m}\right) \in R^{2 m}\right\}, \\
\tilde{u}\left(t, x, y, y_{k+1}, \cdots, y_{m}\right)=u(t, x, y), \\
\tilde{L} v \equiv L v+\sum_{i=k+1}^{m} x_{i} \frac{\partial v}{\partial y_{i}} .
\end{gathered}
$$

It is clear that $\tilde{u} \geqq 0$ and $\tilde{L} \widetilde{u}=0$ in $\tilde{\Pi}$. Also, $\widetilde{u}=0$ if $t=0$. Therefore, if we prove the theorem in case $k=m$ then $\tilde{u} \equiv 0$ in $\tilde{I}$ and, consequently, $u \equiv 0$ in $\Pi$. Thus, it suffices to prove the original theorem in case $u \geqq 0$ in $\Pi$ and $k=m$.

For any $R>0$,

$$
u(t, x, y) \geqq \int_{|\vDash|^{2}+|\eta|^{2}<R^{2}} \Gamma(t, x, y ; \tau, \xi, \eta) u(\tau, \xi, \eta) d \xi d \eta .
$$

Indeed, denoting the right hand side by $v(t, x, y)$, we have: $u-v \geqq 0$ on $t=\tau$ and

$$
\liminf _{|x|+|y| \rightarrow \infty}(u-v) \geqq 0 .
$$

Hence by Theorem 9 in [3; p. 43] (which holds also for degenerate parabolic operators, since the proof requires only the weak form of the maximum principle), $u-v \geqq 0$ in $\Pi$.

Integrating both sides of (4.7) with respect to $\tau, 0 \leqq \tau \leqq t^{*}$, for some $t^{*} \in(0, t)$, and taking $R \rightarrow \infty$, we obtain

$$
\int_{0}^{t^{*}} \int_{R^{m}} \int_{R^{k}} \Gamma(t, x, y ; \tau, \xi, \eta) u(\tau, \xi, \eta) d \xi d \eta d \tau \leqq t^{*} u(t, x, y) .
$$

If $\gamma>c(t, x, y)$ then 


$$
e^{\gamma t} \leqq \int_{R^{m}} \int_{R^{k}} \Gamma(t, x, y ; s, \xi, \eta) e^{\gamma s} d \xi d \eta
$$

Indeed, denoting right hand side by $w(t, x, y)$, we have: $L\left(e^{r t}-w\right)=$ $L e^{r t} \leqq 0, e^{r t}-w=0$ on $t=s, e^{r t}-w$ is bounded in the strip $[s, t]$. Now apply Theorem 9 in [3; p. 43]. From (4.9) with $s=0$ we conclude that, for any $(\bar{t}, \bar{x}, \bar{y})$,

$$
\Gamma\left(\bar{t}, \bar{x}, \bar{y} ; 0, \xi^{0}, \eta^{0}\right)>0 \text { for some }\left(\xi^{0}, \eta^{0}\right) \text {. }
$$

Hence, for some $\rho>0, \alpha>0$,

$$
\Gamma(\bar{t}, \bar{x}, \bar{y} ; \tau, \xi, \eta) \geqq \alpha>0 \text { if } 0 \leqq \tau \leqq t^{*},\left|\xi-\xi^{0}\right|^{2}+\left|\eta-\eta^{0}\right|^{2} \leqq \rho^{2},
$$

provided $t^{*}$ is sufficiently small.

Lemma 2. Under the assumptions of Theorem 6 , for any $(\bar{t}, \bar{x}, \bar{y})$ and $t^{*}, \rho, \xi^{0}, \eta^{0}$ as in (4.10), and for any $0<\hat{t}<t^{*}, \hat{t}$ sufficiently small,

$$
\Gamma(\bar{t}, \bar{x}, \bar{y} ; \tau, \xi, \eta) \geqq c \exp \left\{-\lambda\left[\left|\xi-\xi^{0}\right|^{2}+\left|\eta-\eta^{0}\right|^{2}\right]\right\}
$$

provided $\left|\xi-\xi^{0}\right|^{2}+\left|\eta-\eta^{0}\right|^{2} \geqq \rho^{2}, 0 \leqq \tau \leqq \hat{t}$, where c, $\lambda$ are positive constants.

A similar result holds for nondegenerate parabolic equations; see [3], [1]. The proof given below employs a comparison argument as in [1].

Proof. Take for simplicity $\xi^{0}=0, \eta^{0}=0$. Consider the function

$$
V(\tau, \xi, \eta)=\exp \left\{-\frac{\lambda|\xi|^{2}}{\sigma-\tau}-\frac{\lambda}{(\sigma-\tau)^{3}}|\mu \eta+(\sigma-\tau) \xi|^{2}\right\}
$$

for $0<\tau<\sigma,|\xi|^{2}+|\eta|^{2} \geqq \rho^{2}$, where $\sigma \in\left(0, t^{*}\right)$. If $\sigma$ is sufficiently small and $|\mu|>1$, then

$$
\frac{|\xi|^{2}}{\sigma-\tau}+\frac{|\mu \eta+(\sigma-\tau) \xi|^{2}}{(\sigma-\tau)^{3}} \geqq 1
$$

Writing

$$
L^{*} w=\sum_{i, j=1}^{m} a_{i j} \frac{\partial^{2} w}{\partial \xi_{i} \partial \xi_{j}}+\sum_{i=1}^{m} \widetilde{a}_{i} \frac{\partial w}{\partial \xi_{i}}-\sum_{i=1}^{m} \xi_{i} \frac{\partial w}{\partial \eta_{i}}+\widetilde{c} w+\frac{\partial w}{\partial \tau}
$$

and setting 


$$
\zeta=\frac{\mu \eta+(\sigma-\tau) \xi}{\sigma-\tau}, \quad \tilde{a}=\left(\widetilde{a}_{1}, \cdots, \widetilde{a}_{m}\right)
$$

one easily verifies that

$$
\begin{aligned}
\frac{L^{*} V}{V} \geqq & \frac{4 \lambda^{2} \alpha_{0}}{(\sigma-\tau)^{2}}|\xi+\zeta|^{2}-\left(\Sigma a_{i i}\right) \frac{4 \lambda}{\sigma-\tau}-\frac{2 \lambda}{\sigma-\tau} \tilde{a} \cdot(\xi+\zeta) \\
& +\frac{2 \lambda \mu}{(\sigma-\tau)^{2}} \xi \cdot \zeta-\frac{\lambda|\xi|^{2}}{(\sigma-\tau)^{2}}-\frac{3 \lambda|\zeta|^{2}}{(\sigma-\tau)^{2}}+\frac{2 \lambda}{(\sigma-\tau)^{2}} \xi \cdot \zeta+\widetilde{c} .
\end{aligned}
$$

Writing $|\xi+\zeta|^{2}=|\xi|^{2}+|\zeta|^{2}+2 \xi \cdot \zeta$ and taking $\mu=-\left(4 \alpha_{0} \lambda+1\right)$, the terms involving $\xi \cdot \zeta$ disappear from the right hand side. Taking $\lambda$ sufficiently large and using (4.12), we then conclude that

$$
L^{*} V>0 \text { if } 0 \leqq \tau<\sigma,|\xi|^{2}+|\eta|^{2} \geqq \rho^{2} .
$$

We shall compare $V$ with

$$
w(\tau, \xi, \eta)=\Gamma(\bar{t}, \bar{x}, \bar{y} ; \tau, \xi, \eta)
$$

in the region $0<\tau<\sigma,|\xi|^{2}+|\eta|^{2} \geqq \rho^{2}$. By (4.5), (4.13), $L^{*}(w-\alpha V)<0$ in this region, where $\alpha$ is any positive number. Taking $\alpha$ as in (4.10) we have $w-\alpha V \geqq 0$ if $0 \leqq \tau \leqq \sigma,|\xi|^{2}+|\eta|^{2}=\rho^{2}$. Since also $w-\alpha V=w \geqq 0$ if $\tau=\sigma,|\xi|^{2}+|\eta|^{2} \geqq \rho^{2}$, we can apply Theorem 9 of [3; p. 43] to conclude that $w-\alpha V \geqq 0$ in the region $0<\tau<\sigma$, $|\xi|^{2}+|\eta|^{2} \geqq \rho^{2}$. This yields the assertion of the lemma (with a different $\lambda$ ) for any $0<\hat{t}<\sigma$.

Substituting (4.11) into (4.8) we obtain

$$
\int_{0}^{\hat{t}} \int_{R^{m}} \int_{R^{m}} u(\tau, \xi, \eta) \exp \left\{-\mu\left[|\xi|^{2}+|\eta|^{2}\right]\right\} d \xi d \eta d \tau \leqq C<\infty
$$

where $\mu, C$ are positive constants.

We shall deduce from (4.14) that $u \equiv 0$, employing an argument similar to that used in Lemma 5 of [8]. Let

$$
Z(t, x, y)=(\hat{t}-t) \exp \left\{-\frac{\varepsilon|x|^{2}+\varepsilon|y|^{2}}{1+Q(\hat{t}-t)}\right\} .
$$

For any $\varepsilon>0$,

$$
L^{*} Z<0 \text { if } 0<t<\hat{t}, x \in R^{m}, y \in R^{m}
$$

provided $\hat{t}$ is sufficiently small and $Q$ is sufficiently large. Let $\zeta(x, y)$ be a $C^{\infty}$ function satisfying $\zeta(x, y)=1$ if $|x|^{2}+|y|^{2}<R^{2}$, $\zeta(x, y)=0$ if $|x|^{2}+|y|^{2}>R^{2}+1,0 \leqq \zeta(x, y) \leqq 1$ elsewhere. We can choose $\zeta$ so that its first derivatives are bounded by a constant independent of $R$. Let 


$$
\hat{Z}(t, x, y)=\zeta(x, y) Z(t, x, y) .
$$

Then, by Green's identity,

$$
\int_{0}^{\hat{t}} \int_{R^{2 m}}\left(L^{*} \hat{Z} \cdot u-\hat{Z} L u\right) d x d y d t=0 .
$$

We have used here the facts that $\hat{Z}(\hat{t}, x, y)=0, u(0, x, y)=0$. Since $L u=0$, we get

$$
\int_{0}^{\hat{t}} \int_{|x|^{2}+|y|^{2}<R^{2}} L^{*} Z \cdot u d x d y d t=-\int_{0}^{\hat{t}} \int_{R^{2}<|x|^{2}+|y|^{2}<R^{2}+1} L^{*} \hat{Z} \cdot u d x d y d t .
$$

Taking $\varepsilon>\mu$ and using (4.14) one easily concludes that the right hand side converges to 0 if $R \rightarrow \infty$. Since $u \geqq 0, L^{*} Z<0$, it follows that $u(t, x, y) \equiv 0$ in the strip $0 \leqq t \leqq \hat{t}$. Now proceed step by step to show that $u \equiv 0$ in the strip $0 \leqq t \leqq T$.

REMARK. Denote by $\tilde{L} u$ the operator obtained from $L u$ in (4.1) upon replacing

$$
\sum_{i=1}^{k} x_{i} \frac{\partial u}{\partial y_{i}} \text { by } \sum_{i=1}^{k} b_{i}\left(t, x^{\prime}, y\right) \frac{\partial u}{\partial y_{i}}, x^{\prime}=\left(x_{1}, \cdots, x_{k}\right) .
$$

Suppose the transformation

$$
\bar{x}_{i}=b_{i}\left(t, x^{\prime}, y\right) \quad(i=1, \cdots, k)
$$

is a diffeomorphism from $R^{k}$ onto $R^{k}$ and that the first four derivatives of this mapping and of its inverse are bounded. Then, by the change of variables (4.15) we obtain an operator of the form (4.1) to which Theorem 6 can be applied. Consequently, Theorem 6 extends also to the operator $\widetilde{L}$.

\section{REFERENCES}

1. A. G. Aronson and P. Besala, Uniqueness of positive solutions of parabolic equations with unbounded coefficients, Colloq. Math., 18 (1967), 125-135.

2. M. I. Freidlin, On the factorization of nonnegative definite matrices, Theory Probability App!., 13 (1968), 354-356 [Teor. Verjatnost.; Primenen. 13 (1968), 375-378].

3. A. Friedman, Partial Differential Equations of Parabolic Type, Prentice-Hall, Englewood Cliffs, N. J., 1964.

4. T. Genčev, The Cauchy problem for a class of ultraparabolic equations, Annuaire Univ. Sofia, Fac. Math., 58 (1963/64) 141-169.

5. I. I. Gikhman and A. V. Skorokhod, Introduction to the Theory of Random Processes, W. B. Saunders, Philadelphia, 1969.

6. —_ Stochastic Differential Equations, "Naukova Dumka” Kiev, 1968.

7. A. M. Ilin, On a class of ultraparabolic equations, Soviet Math., 5 (1964), 16731676. [Doklady Akad. Nauk SSSR, 159 (1964), 1214-1217].

8. V. A Kondratév and S. D. Eidelman, Properties of solutions of linear evolutionary 
systems with elliptic space part, Math. USSR Sbornik, 10 (1970), 369-397. [Mat. Sbornik, 81 (123) (1970), no. 3.J

9. R. S. Phillips and L. Sarason, Elliptic-parabolic equations of the second order, J. Math. Mach., 17 (1968), 891-917.

10. G. N. Smirnov, The Cauchy problem for parabolic equations degenerating at infinity, Math. Sbornik, 70 (112) (1966), 591-604.

11. I. M. Sonin, On a class of degenerate diffusion processes, Theory Prob. Appl., 12 (1967), 490-496.

12. On uniqueness class for degenerate parabolic equations, Math. Sbornik, 87 (127) (1971), 459-473.

13. M. Weber, The fundamental solution of a degenerate partial differential equation, Trans. Amer. Math. Soc., 71 (1951), 24-34.

Received February 18, 1972. This work was partially supported by National Science Foundation grant GP-28484.

NORTHWESTERN UNIVERSITY 



\section{PACIFIC JOURNAL OF MATHEMATICS}

\section{EDITORS}

D. Gilbarg and J. Milgram Stanford University

Stanford, California 94305

R. A. Beaumont

University of Washington

Seattle, Washington 98105
J. DugundJI

Department of Mathematics

University of Southern California

Los Angeles, California 90007

RICHARD ARENS

University of California

Los Angeles, California 90024

ASSOCIATE EDITORS
E. F. BECKENBACH
B. H. NeumanN
F. WOLF
K. YoSHIDA

\section{SUPPORTING INSTITUTIONS}

UNIVERSITY OF BRITISH COLUMBIA

CALIFORNIA INSTITUTE OF TECHNOLOGY

UNIVERSITY OF CALIFORNIA

MONTANA STATE UNIVERSITY

UNIVERSITY OF NEVADA

NEW MEXICO STATE UNIVERSITY

OREGON STATE UNIVERSITY

UNIVERSITY OF OREGON

OSAKA UNIVERSITY
UNIVERSITY OF SOUTHERN CALIFORNIA

STANFORD UNIVERSITY

UNIVERSITY OF TOKYO

UNIVERSITY OF UTAH

WASHINGTON STATE UNIVERSITY

UNIVERSITY OF WASHINGTON

$\stackrel{*}{*} \stackrel{*}{*} \stackrel{*}{*}$ AMERICAN MATHEMATICAL SOCIETY

NAVAL WEAPONS CENTER 


\section{Pacific Journal of Mathematics}

\section{Vol. 46, No. $1 \quad$ November, 1973}

Allan Francis Abrahamse, Uniform integrability of derivatives on $\sigma$-lattices .......................................... 1

Ronald Alter and K. K. Kubota, The diophantine equation $x^{2}+D=p^{n} \ldots \ldots \quad 11$

Grahame Bennett, Some inclusion theorems for sequence spaces .......... 17

William Cutler, On extending isotopies ........................ 31

Robert Jay Daverman, Factored codimension one cells in Euclidean

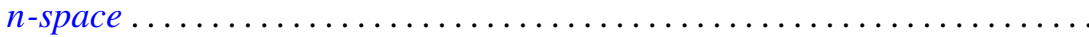

Patrick Barry Eberlein and Barrett O’Neill, Visibility manifolds ............ 45

M. Edelstein, Concerning dentability .......................... 111

Edward Graham Evans, Jr., Krull-Schmidt and cancellation over local

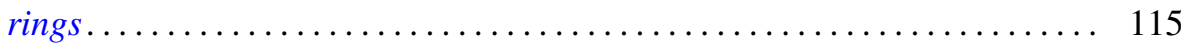

C. D. Feustel, A generalization of Kneser's conjecture ................ 123

Avner Friedman, Uniqueness for the Cauchy problem for degenerate parabolic equations .......................................... 131

David Golber, The cohomological description of a torus action ............ 149

Alain Goullet de Rugy, Un théorème du genre "Andô-Edwards" pour les

Fréchet ordonnés normaux..............................

Louise Hay, The class of recursively enumerable subsets of a recursively enumerable set ........................................

John Paul Helm, Albert Ronald da Silva Meyer and Paul Ruel Young, On orders of translations and enumerations...

Julien O. Hennefeld, A decomposition for $B(X)^{*}$ and unique Hahn-Banach

extensions

Gordon G. Johnson, Moment sequences in Hilbert space .

Thomas Rollin Kramer, A note on countably subparacompact spaces ...

Yves A. Lequain, Differential simplicity and extensions of a derivation ....

Peter Lorimer, A property of the groups Aut $\mathrm{PU}\left(3, q^{2}\right) \ldots$

225

Yasou Matsugu, The Levi problem for a product manifold.

231

John M.F. O'Connell, Real parts of uniform algebras ......

William Lindall Paschke, A factorable Banach algebra without bounded

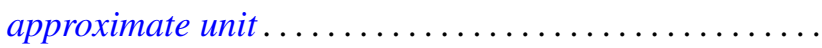

Ronald Joel Rudman, On the fundamental unit of a purely cubic field ....

Tsuan Wu Ting, Torsional rigidities in the elastic-plastic torsion of simply connected cylindrical bars .........................

Philip C. Tonne, Matrix representations for linear transformations on analytic sequences...................................

Jung-Hsien Tsai, On E-compact spaces and generalizations of perfect

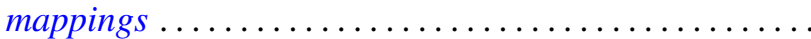

Alfons Van Daele, The upper envelope of invariant functionals majorized by an invariant weight. .. 